\title{
Wake Vortices Behavior Near the Ground
}

\author{
Alexandre Corjon* and Thierry Poinsot \\ Department of Fluid Mechanics \\ CERFACS, 42 G. Coriolis Ave, F-31057 \\ Toulouse Cedex, France
}

\begin{abstract}
Direct numerical simulations of a vortex pair embedded in a stable atmospheric boundary layer are presented. The effects of various crosswind conditions are studied. These computations demonstrate the creation of secondary vortices for the range of Reynolds numbers $\left(3770 \leq R e_{\Gamma} \leq\right.$ 7540). The physics of wake vortex interactions with the ground for different values of crosswind are discussed. The redistribution of vorticity between the atmospheric boundary layer and the vorticity induced by the primary vortices may explain the vortex tilting phenomenon. A parametrization of the effect of crosswind on the minimum altitude reached by the two vortices is given.
\end{abstract}

\section{Introduction}

With the growth of air traffic and the design of new high capacity aircrafts, problems related to wake vortices are becoming more and more important. Throughout the world, new studies on these problems are being initiated. The regulatory authorities are interested in developing air traffic systems aimed at safely reducing the spacing between aircraft during landing and take-off phases to increase airport capacity. The aircraft manufacturers have a rested interest in alleviating the wake vortices to avoid categorization problems which lead to less efficient high capacity aircrafts. For this reason, they need to have a better understanding of the wake vortices behavior near the ground for given meteorological conditions. This knowledge has to be implemented in an engineering model with real time capabilities in order to determine the hazards associated with these vortices (position and strength). CERFACS is developing such an operational model for the STNA (Service Technique de la Navigation Aérienne). The development of this engineering model is based both on experimental results collected on airports and numerical simulations. The hazard is more severe near the ground because of the shorter recovery time available. The Civil Aviation Authority in England [7] has shown that the greatest frequency of reported incidents occurs to following aircrafts in the height band of $100 \mathrm{ft}$ to $200 \mathrm{ft}$ where the trailing vortices are affected by the ground. This could be explained by the fact that all aircraft follow the same flight path while vortices may bounce on the ground. Therefore, the behavior of the wake vortices depends on the velocity of the crosswind.

The work reported here is focused on the laminar viscous effect of the ground on the trailing vortex trajectories with crosswind. A first version of an engineering model, called VORTEX [5], is based on Greene's model [9] for stratification and atmospheric turbulence effects and it includes the effects of the ground and crosswind. This model assumes that the vortex sheet shed by the aircraft

${ }^{*}$ Consultant for STNA, 246 rue Lecourbe, 75732 PARIS Cedex 15 (France) 
has completely rolled-up and that only two counter-rotating vortices remain in the atmosphere. As in the model first presented by Tombach [18], the vorticity is assumed to be concentrated into the two counter-rotating vortex cores whose diameters are small compared to their spacing, so that the movement of two point vortices could be considered. The transport of the trailing vortices is modeled by the laminar potential flow solution of infinite line vortices. This solution indicates that, without the effect of the ground, the two vortices will descend while maintaining a constant distance ( $d_{0}$ the initial spacing). The first effect of the ground plane is to make the pair diverge. This divergence is due to an inviscid phenomenon. The ground plane may be modeled by two image vortices whose strengths are equal and opposite to those of the real vortices in order to satisfy the slip boundary condition in the inviscid theory.

When the real vortex and its image are close enough, their mutual interaction has a greater importance than the interaction of the two real vortices. This behavior could be expressed analytically [17] and the minimum altitude reached by the vortices is half of their initial spacing $d_{0}$.

The second effect of the ground is a viscous effect. Experiments $[8,10]$ show that the trajectory of the vortices near the ground in the atmosphere differs from the inviscid trajectory. Barker and Crow [1] asserted that the rebound was due to the finite vortex core size but Saffman [17] showed that the bouncing of the vortices could not be explained in the framework of inviscid theory. The explanation proposed by Harvey and Perry (and which was confirmed by many theoretical studies $[4,14,15])$ is based on viscous phenomena at the wall and is illustrated in Fig. 1. During their descent, each primary vortex induces a boundary layer. As they continue to descend this vorticity sheet detaches from the wall thus creating a secondary vortex. This secondary vortex rolls over the primary and makes it rise up.

In VORTEX [5], classical two-dimensional inviscid theory is modified as proposed by Liu [14] to introduce vortex rebound. A line vortex is added near the wall to model the effect of the secondary vortex. This method introduces three new parameters in VORTEX which are:

- $r_{i}^{\prime}=d_{0} / 2$ : the constant distance between the primary vortex $i$ and its secondary vortex. $d_{0}$ is the initial spacing between the two primary vortices. $r_{i}^{\prime}$ is supposed to be constant $[6,14]$.

- $\Gamma_{i}^{\prime}$ : the circulation of the secondary vortex

- $h_{0}$ : altitude of rebound.

The altitude of rebound $h_{0}$ is in fact the altitude that must be reached by the primary vortex to introduce the secondary vortex near the ground. In [5], it is shown that if the two first parameters are well defined, the third (the altitude $h_{0}$ ) depends on the value of the crosswind. The following work presents two-dimensional, unsteady direct numerical simulations of vortex rebound with and without crosswind. The physics of vortex rebound and the influence of crosswind on the minimum altitude reached by the wake vortices are described, and the tentative parametrization of $h_{0}$ as a function of crosswind shear is proposed.

\section{Methodology}

For the direct numerical simulations (DNS), we use a Navier-Stokes solver available at CERFACS, called NTMIX [2]. The aims of these computations are not only to validate the engineering model but also to set the values of VORTEX parameters. As said before, here we concentrate on the altitude of rebound.

The numerical scheme is given in Lele [12] and Poinsot and Lele [16]. Here we illustrate only its main features. This code was developed first to compute flows with complex chemistry to study 
combustion phenomena. NTMIX solves the fully compressible two-dimensional Navier-Stokes equations. For the spatial differentiation, high-order $\left(6^{\text {th }}\right)$ compact schemes of spectral-like resolution are used and for time integration, a third order Runge-Kutta method. The boundary conditions are defined using the NSCBC method presented in [16]. For the present work, inlet, outlet and top boundaries are non reflecting: convective and acoustic waves propagate through them with negligible reflection.

For the initialization, Lamb's vortex are used whose streamfunction is:

$$
\psi=-g \ln \left(r^{2}+r_{c}^{2}\right) \Longrightarrow\left\{u_{r}=0 / / u_{\theta}(r)=\frac{\Gamma}{2 \pi} \frac{r}{r^{2}+r_{c}^{2}}\right.
$$

where $g$ is related to the vortex circulation $\Gamma=4 \pi g, r_{c}$ is the core radius and $r$ the distance from the vortex center. The Reynolds number of the computation is:

$$
R e=\frac{u_{\theta_{\max }} r_{c}}{\nu}=\frac{\Gamma}{4 \pi \nu}
$$

where $u_{\theta_{\max }}$ is the maximal tangential velocity reached at $r=r_{c}$.

The initialization is made with 4 vortices: two real vortices and their mirror images to ensure a zero normal velocity at the wall (the no-slip condition is verified by imposing a zero parallel velocity at the wall). The computation domain is presented on Fig. 2.

The boundary conditions are:

- inlet: subsonic inlet or non reflecting

$$
u=U(y)=\frac{u_{f}}{\kappa} \log \left(\frac{y}{y_{r}}\right) \text { or } u=U(y)=\frac{U\left(y_{\max }\right)}{\log \left(\frac{y_{\max }}{y_{r}}\right)} \log \left(\frac{y}{y_{r}}\right)
$$

- outlet: non reflecting (convective and acoustic waves exit with negligible reflection)

- bottom: no-slip wall

- top: non reflecting

where $u_{f}$ is the friction velocity at the wall, $\kappa=0.4$ the Von-Karman constant and $y_{r}$ the height of roughness and $y_{\max }$ the height of the computational domain. The inlet wind profile is given by Monin-Obukhov similarity theory for the surface boundary layer [13]. It corresponds to neutral condition in the surface boundary layer $(y \leq 100 \mathrm{~m})$. All lengths are normalized using the wingspan $b$. The initial spacing $b_{0}$ is set to $0.8 b$ which is the same as for a wing with an elliptical loading [3] $\left(b_{0}=(\pi / 4) b\right)$. The domain size for all the computations is $\left(0<x^{*}<6,0<y^{*}<2\right)$.

The results are presented using a dimensionless time $t^{\prime \prime}$ equal to $u_{\theta_{\max }} t / b_{0}$. Velocities will be scaled using the speed $c$ and the computation Reynolds number is $R e_{\text {ref }}=c b / \nu$ (dimensionless variables using this normalization are denoted with ${ }^{*}$ ). In all the test-cases with crosswind, the grid used is $363 \times 121$ points except for the test-case without crosswind, where the grid is $257 \times 121$ points. The vortices are initially put at $\left(x_{\text {med }}^{*} \pm 0.4\right.$ and $\left.y^{*}=1\right)$.

\section{Results}

\subsection{Iso-contours of vorticity}

Table 1 presents the parameters of all the test cases. The first results presented in Fig. 3 are the results obtained without crosswind $\left(U^{*}=0.00\right)$ at four different times: $t^{\prime \prime}=0.00,5.38,8.03$ and 
15.05 (see Table 1, Case 1). The initial conditions are: $g^{*}=g /(b c)=0.03, r_{c}^{*}=r_{c} / b=0.1$ (core radius), $b_{0}^{*}=b_{0} / b=0.8$ and $x_{\text {med }}^{*}=0$. The vortex Reynolds number $R e=u_{\theta_{\max }} r_{c} / \nu$ is equal to 600. The iso-contours of vorticity $\omega^{*}=\omega b / c$ are drawn from -5 . to 5 . with a step $\Delta \omega=0.5$.

The rebound mechanism is in agreement with the explanation of Harvey and Perry [10]. The vortex creates a wall boundary layer during its descent with a strong vorticity sheet. This vorticity sheet is elongated until it breaks and creates a secondary vortex. This vortex rolls over the primary one and makes it rise up away from the wall. Then, if only the trajectory of the main vortex is considered, the primary vortex bounces on the wall. These results have been compared [5] to those of Orlandi [15] and are in good agreement even if the Reynolds number of the computations are not exactly the same $(R e=800$ for Orlandi).

Figures 4 to 6 present the results obtained for three values of crosswind: $U_{\max }^{*}=0.05$ (Case 2), 0.10 (Case 3) and 0.20 (Case 4). The initial conditions of the computations are the same for the vortices: $g^{*}=0.03, r_{c}^{*}=0.1$ and $b_{0}^{*}=0.8$. The maximal value of the logarithmic profile of crosswind is different (see above) and also the value of $x_{\text {med }}^{*}\left(x_{\text {med }}^{*}=2\right.$. for $U_{\text {max }}^{*}=0.05$ and $x_{\text {med }}^{*}=1.4$ for the two other values). Figure 4 (Case 2) presents the evolution of vorticity at four instants: $t^{\prime \prime}=0.00,4.50,6.75$ and 11.25. The observed mechanism is the same as in Fig. 3. Due to their mutual interaction, the two primary vortices descend and create a strong vorticity sheet. As the vortices move down, due to the adverse pressure gradient, the boundary layers separate, creating the secondary vortices.

The main difference with the case without crosswind is the asymmetry of the upwind (left) and downwind (right) vortex pair. The vorticity of the downwind secondary vortex has the same sign as the crosswind and the separation is favored. On the contrary, the separation is delayed for the upwind secondary vortex with the opposite sign of vorticity due to the crosswind.

Figure 5 (Case 3 ) shows the contours of vorticity in the case of a crosswind equal to $U_{\max }^{*}=0.10$ are presented. The times are the same as in Fig. 4 as are the values of the contours. In this case, the velocity of the crosswind is $1 / 3$ of the maximal tangential velocity $u_{\theta_{\max }}$ of the vortices (see Table 1). The behavior of the vortices described before is more obvious here. The asymmetry between the upwind and downwind secondary vortex is amplified and at $t^{\prime \prime}=11.25$, as the downwind pair is moving away from the wall, the secondary vortex for the upwind pair has not yet detached. At this time, a tertiary vortex with the same sign of vorticity as the primary is also observed near the upwind vortex.

Finally, Fig. 6 (Case 4) shows the evolution of the vorticity for a crosswind of $U_{\max }^{*}=0.20$. For this case, the crosswind is high (2/3 of the maximal tangential velocity of the vortices). At $t^{\prime \prime}=6.75$, the secondary vortex for the upwind vortex is not created. The opposite vorticity due to crosswind is able to counteract the effect of the descending upwind primary vortex.

If we examine the separation for the downwind vortex (Fig. 7) and the upwind one (Fig. 8), the effect of the crosswind on the boundary layers induced by the primary vortices may be observed: all snapshots are made at the same time, $t^{\prime \prime}=4.5$, and illustrate differences in the evolution of the vorticity near the ground. For the downwind pair (Fig. 7), the trace of the tertiary vortex can be seen, just below the secondary vortex. This trace is less obvious as the crosswind speed increases. When the crosswind velocity increases, separation occurs earlier. For $U_{\max }^{*}=0.20$ (Fig. 7 (d)), the vorticity sheet is more elongated and the primary vortex is at higher altitude than in the other cases.

For the downwind pair, Fig. 8 (a) is the same as for Fig. 7 (a), because the computation is symmetrical when there is no crosswind. It is obvious that the vorticity induced by the crosswind, which is more important near the ground plane, counteracts the creation of the secondary vortex. For a crosswind of $U_{\max }^{*}=0.20($ Fig. $8(\mathrm{~d})$ ), the rebound of the upwind vortex will certainly never occur. 


\subsection{Trajectories}

The previous results are summarized in Fig. 9 which displays the trajectories of the primary vortices. The first remark is that the altitude of rebound increases with the crosswind velocity.

In the same way, the length of rebound (the distance along the x-axis during which the vortices are in a ground effect) increases with the crosswind. The primary-secondary vortex pair is advected by the crosswind. From an operational point of view the case with the milder crosswind $\left(U_{\max }^{*}=\right.$ $0.05)$ is the most hazardous case. The upwind vortex stays near the runway axis and may cause trouble to the following aircraft. In all other cases, the crosswind sweeps the upwind and downwind vortex from the runway and there is no more hazard for a following aircraft.

A useful dimensionless number to study trajectories is $W_{S}=U_{0} b_{0} / \Gamma_{0}$ [5], where $U_{0}$ is the value of crosswind at initial altitude, $b_{0}$ the initial spacing between the vortices and $\Gamma_{0}$ their initial circulation. However, the minimum altitude reached by the vortices does not correlate with $W_{S}$ (see Fig. 10). In Fig. 10, $h_{\min }^{o}$ stands for the minimum altitude reached by the vortices without crosswind, $h_{\min }$ for this minimum altitude in the considered case and then the difference between these two altitudes made dimensionless with the initial spacing is plotted versus $W_{S}$. Six cases are plotted, the four presented above $\left(g^{*}=0.03, b_{0}=0.8 b\right.$ for four values of crosswind and then four values of $W_{S}$ ), plus two other cases where the initial spacing between the vortices is reduced to $b_{0}=0.6 b$ for two crosswind values: $U_{\max }^{*}=0.10$ (Case 5 ) and 0.20 (Case 6 ) (then two new values of $W_{S}$ ). The trend followed by the results varies with the initial spacing between the vortices even if the rebound mechanism is the same as described above.

If the inviscid theory is considered, the minimum altitude reached by the vortices is half of their initial distance. Far from the wall, the vortex pair propagates with a constant vortex spacing $d_{0}$ (which we will call the "reference" spacing) which is the relevant parameter for initial conditions. However, because of resolution requirements, the vortex pair is sometimes initialized at an altitude $y_{0}$ and a vortex spacing $b_{0}$ such that the influence of the wall is already felt.

The reference spacing $d_{0}$ may be deduced from $y_{0}$ and $b_{0}$ using the vortex trajectory in the inviscid theory [11]:

$$
\left(\frac{d_{0}}{2}\right)^{2}\left(x^{2}+y^{2}\right)=x^{2} y^{2}
$$

so that the reference vortex spacing $d_{0}$ is given by:

$$
d_{0}=\frac{2 b_{0} y_{0}}{\sqrt{b_{0}^{2}+4 y_{0}^{2}}}
$$

We will use this length $d_{0}$ to normalize the altitude of rebound. If the wind profile is "physically" the same in these computations, the vorticity experienced by the vortices at $d_{0} / 2$ is different in these two cases. As the vorticity due to crosswind increases near the ground, the shear seen by the vortices in the case of $b_{0}=0.6 b$ (or $d_{0}=0.56 b$ ) is much more important than the shear experienced by the vortices in the case $b_{0}=0.8 b$ (or $d_{0}=0.74 b$ ) for the same maximal value of crosswind.

To describe the effect of crosswind on the vortex bouncing, it is better to use the shear crosswind at an altitude of half the initial distance between the vortices. In the previous paper [5], we showed that the altitude of rebound depends on core radius and initial strength of the vortices, and that we could form a new dimensionless number in order to try to correlate the results:

$$
w_{s}=\frac{\sigma r_{c}}{u_{\theta_{\max }}}
$$

where $\sigma=\left.\frac{d U}{d y}\right|_{y=d_{0} / 2}$ is the shear of the crosswind at $d_{0} / 2$. All the previous results plus three 
others test cases are plotted in Fig. 11 . These other cases are: $g^{*}=0.02$ for two values of crosswind $U_{\max }^{*}=0.05$ (Case 7 ) and 0.10 (Case 8), and $g^{*}=0.015$ for a crosswind $U_{\max }^{*}=0.05$ (Case 9 ).

The correlation between the results is very good for the downwind vortex but relatively poor for the upwind one. The error bars plotted in this Figure indicate the minimum altitude in the computation. It is assumed that this position could vary by \pm 1 grid point and gives an error of \pm 0.022 relatively to $d_{0}$ in the case of $d_{0}=0.74 b$ and \pm 0.029 in the case of $d_{0}=0.56 b$. The linear regression obtained with these results are also plotted in Fig. 11. The regression coefficient is 0.995 for the downwind vortex and -0.907 for the upwind one. To compute the linear regression for the upwind vortex, two points are not taken into account: $g^{*}=0.03$ for $U_{\max }^{*}=0.20$ and $g^{*}=0.02$ for $U_{\max }^{*}=0.10$ because the downwind vortex leaves the computation domain at early times. These results lead to the following formulae:

- for the downwind vortex

$$
\frac{h_{\min }-h_{\min }^{0}}{d_{0}}=2.64 w_{s}
$$

- for the upwind vortex

$$
\frac{h_{\min }-h_{\min }^{0}}{d_{0}}=-1.61 w_{s}
$$

\section{Conclusions}

Using direct numerical simulations, this study shows the effect of crosswind on vortex rebound. The main effect of the crosswind is to asymmetrize the rebound: the downwind vortex bounces earlier than the upwind one. This is due to the effect of crosswind shear which has, in one case, the same sign and in the other, the opposite sign as the secondary vortex created near the wall by the primary one. In a high crosswind, and thus a high shear, the upwind vortex cannot even rebound and follow the inviscid trajectory. These computations show that the experimentaly observed vortex tilting in a crosswind can be explained by the difference in rebound height between the two vortices. Finally, these computations identify a new dimensionless parameter $w_{s}$ which gives the altitude of rebound as function of crosswind and vortices characteristics. This parameter can be used in the engineering model VORTEX to dynamically adjust the rebound height.

\section{Acknowledgments}

This work was supported by the STNA (Service Technique Navigation Aérienne) under research grant STNA/95/110. The authors would like to thank the CEA Saclay and CNUSC for access to their IBM SP2. The authors would also like to thank Dr. M. Baum for NTMIX developments and Mr. C. Le Roux (STNA-3ED) for his help.

\section{References}

[1] Barker, S.J. and Crow, S.C., "The Motion of Two-Dimensional Vortex Pairs in a Ground Effect," J. Fluid Mech., 82 (4), pp. 659-671, 1977

[2] Baum, M., Étude de l'allumage et de la structure des flammes turbulentes, Ph.D. Thesis, École Centrale Paris, 1994.

[3] Betz, A., "Verhaltern von Wirbelsystemen," ZAMM, XII (3), pp. 164-174, 1932. 
[4] Bilanin, A.J., Teske, M.E. and Hirsh, J.E., "Neutral Atmospheric Effects on the Dissipation of Aircraft Vortex Wakes," AIAA J., 16 (9), pp. 956-961, 1978.

[5] Corjon, A. and Poinsot, T., "A Model to define Aircraft Separations due to Wake Vortex Encounter," Proc. 13th AIAA Appl. Aero. Conf., San Diego CA, number AIAA-95-1776-CP, pp. 117-124, June 19-22, 1995.

[6] Corjon, A. and Poinsot, T., "Vortex Model to define Safe Aircraft Separations Distances," J. Aircraft, 33 (3), pp. 547-553, 1996.

[7] Critchley, J.B. and Foot, P.B., "UK CAA Wake Vortex Database: Analysis of Incidents reported between 1982 and 1990," Technical Report PAPER-91, CAA, 1991.

[8] Dee, F.W. and Nicholas, O.P., "Flight Measurements of Wing Tip Vortex Motion near the Ground," Technical Report 68007, RAE, 1968.

[9] Greene, G.C., "An Approximate Model of Vortex Decay in the Atmosphere," J. Aircraft, 23 (7), pp. 566-573, 1986.

[10] Harvey, J.K. and F.J. Perry, "Flowfield produced by Trailing Vortices in the vicinity of the Ground," AIAA J., 9 (8), pp. 1659-1660, 1971.

[11] Lamb, H., Hydrodynamics, Dover publications, New York, 1932.

[12] Lele, S.K., "Compact Finite Difference Schemes with Spectral-like Resolution," J. Comp. Phys., 103, pp. 16-42, 1992.

[13] Lissaman, P.B.S., Crow, S.C., MacCready, P.B., Tombach, I.H. and Bate, E.R., "Aircraft Vortex Wake Descent and Decay under real Atmospheric Effects," Technical Report FAA-RD-73-120, DOT, October 1973.

[14] Liu, H.T., "Tow-Tank Simulation of Vortex Wake Dynamics," FAA Proc. Aircraft Wake Vortices Conf., pp. 32-1,32-26, October 29-31, 1991.

[15] Orlandi, P, "Rebound of Vortex Dipole," Phys. Fluids A, 2 (8), pp. 1429-1436, 1990.

[16] Poinsot, T.J. and Lele, S.K., "Boundary Conditions for direct Simulations of Compressible Viscous Flows," J. Comp. Phys., 101 (1), pp. 104-129, 1992.

[17] Saffman, P.G., "The Approach of a Vortex Pair to a Plane Surace in Inviscid Fluid," J. Fluid Mech., 92, pp. 497-503, 1979.

[18] Tombach, I., "Observations of Atmospheric Effects on Vortex Wake Behavior," J. Aircraft, 10 (11), pp. 641-647, 1973. 


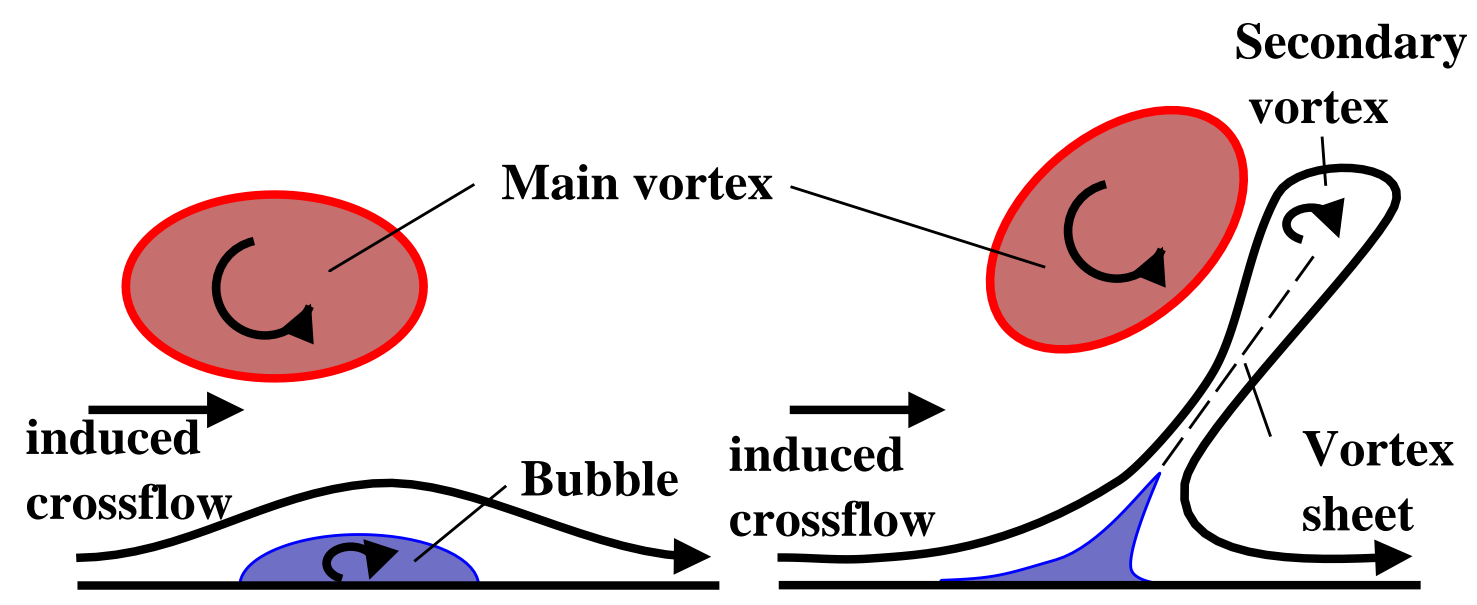

Figure 1: Computational domain.

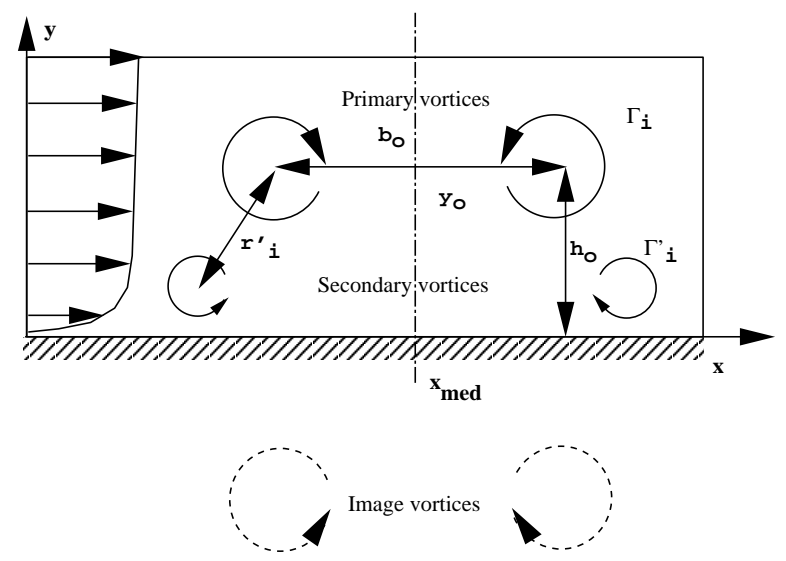

Figure 2: Computation domain. 

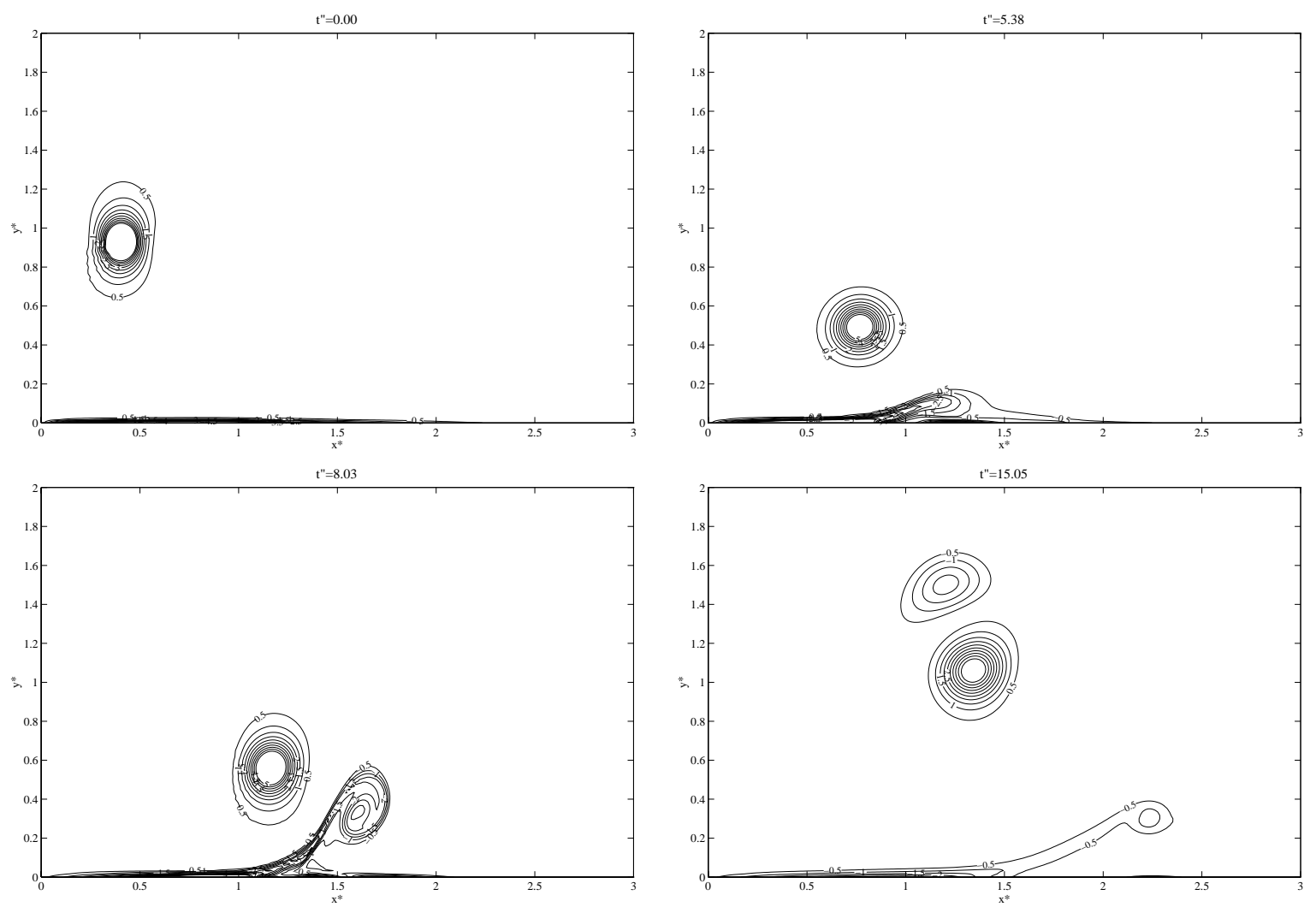

Figure 3: Iso-contours of vorticity - $U^{*}=0.00$. 

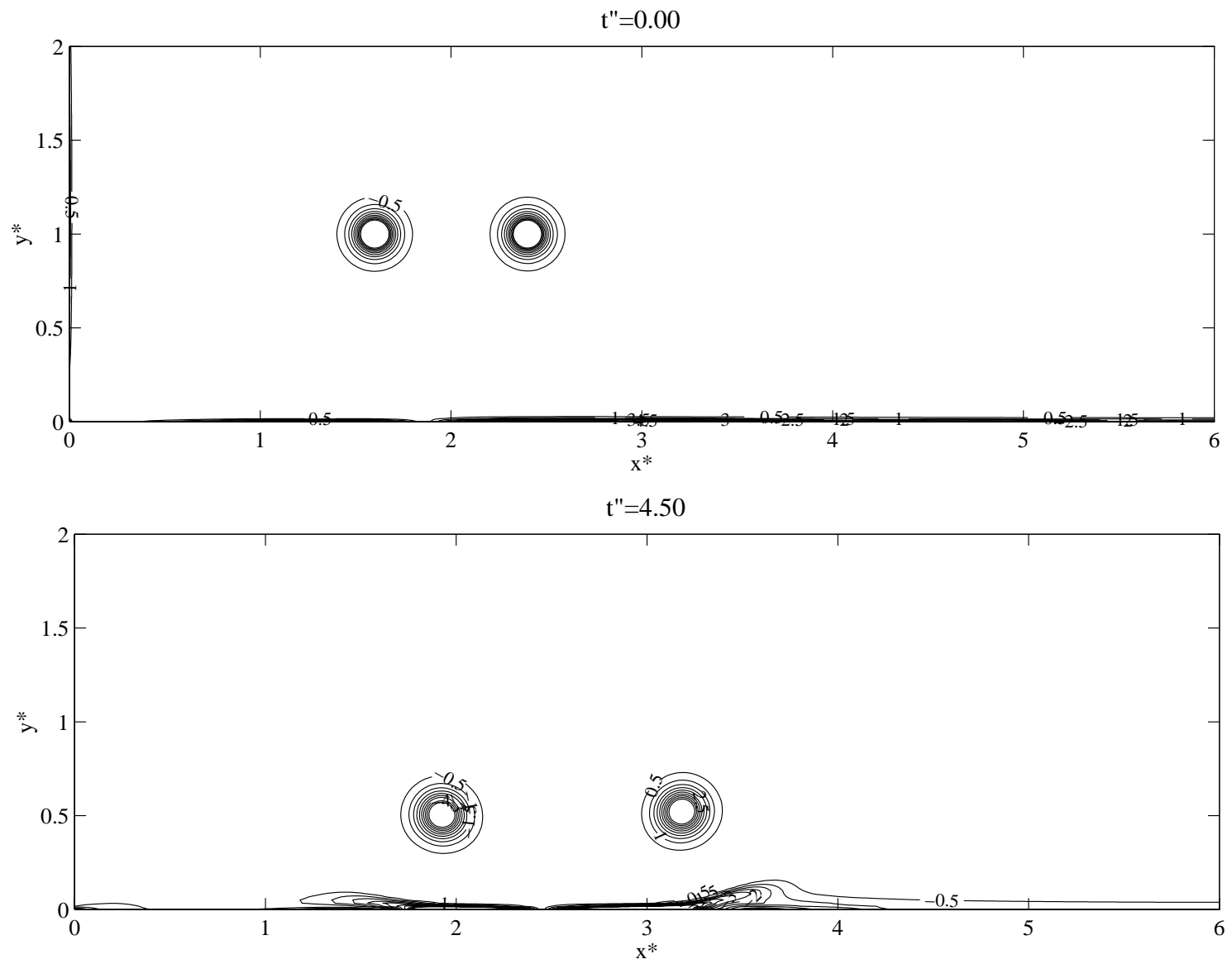

$t^{\prime \prime}=6.75$
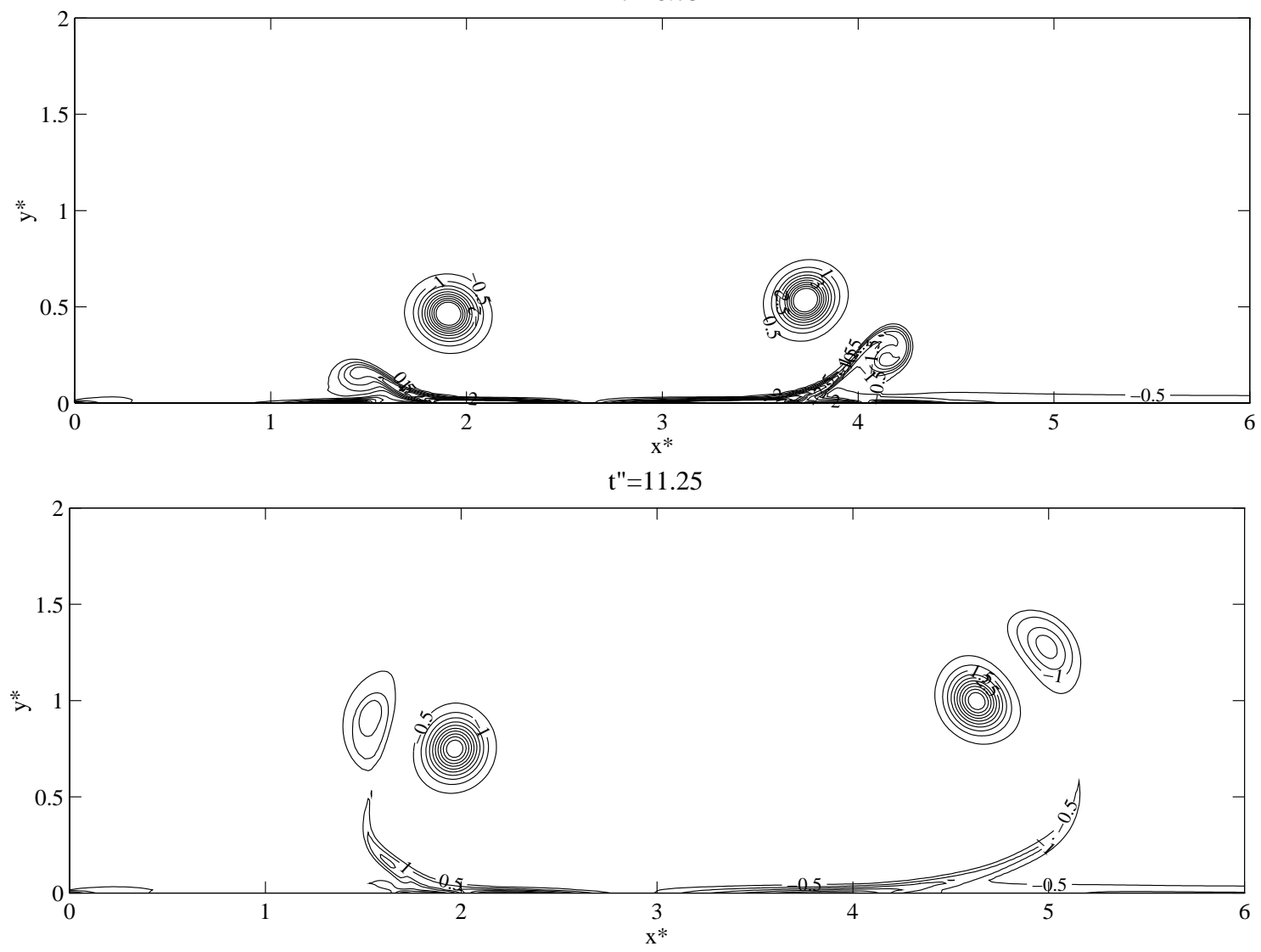

Figure 4: Iso-contours of vorticity - $U_{\max }^{*}=0.05$. 

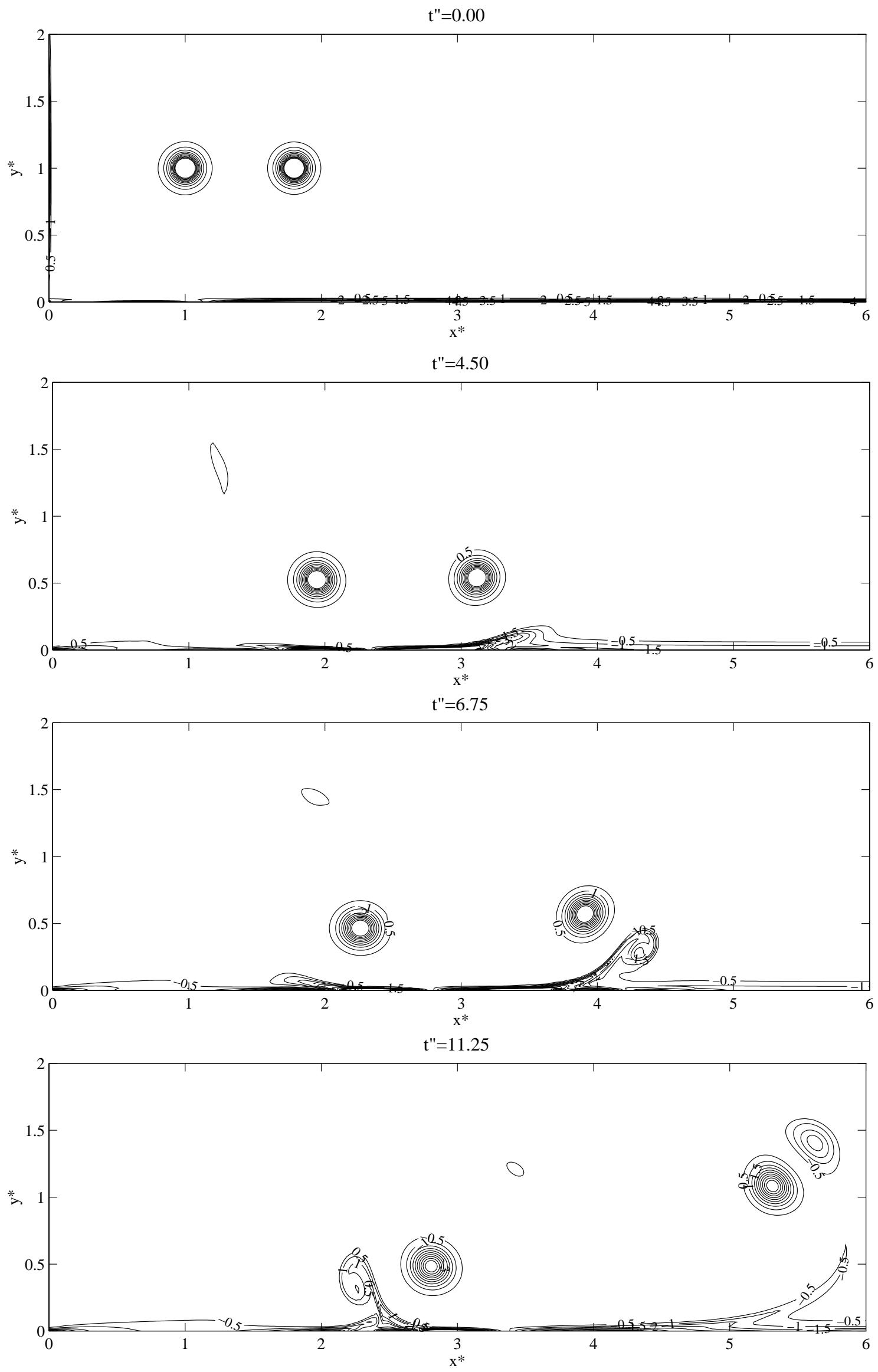

Figure 5: Iso-contours of vorticity - $U_{\max }^{*}=0.10$. 

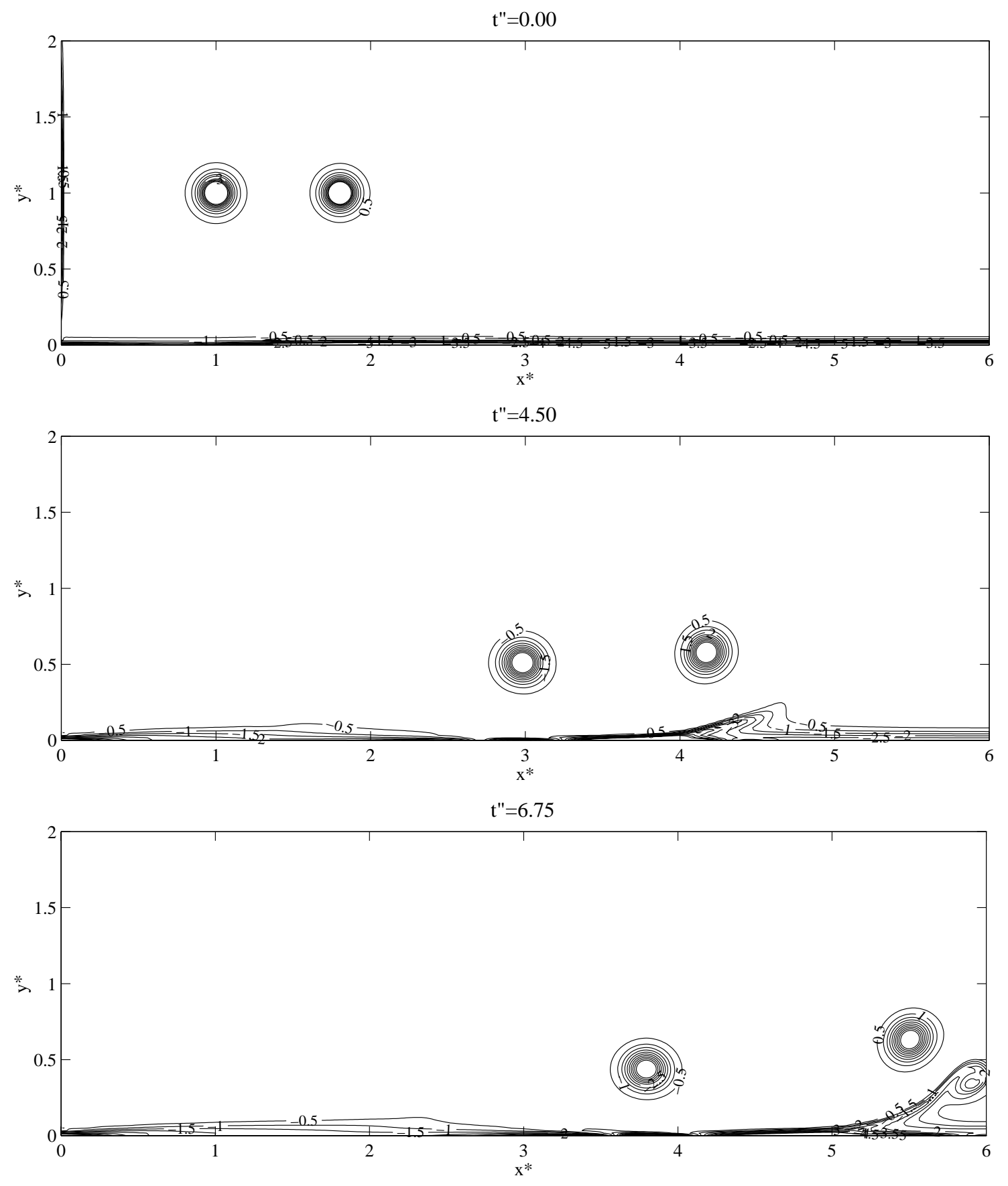

Figure 6: Iso-contours of vorticity - $U_{\max }^{*}=0.20$. 


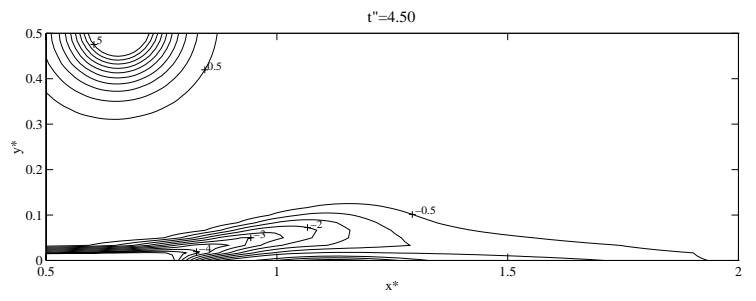

(a) $U_{\max }^{*}=0$

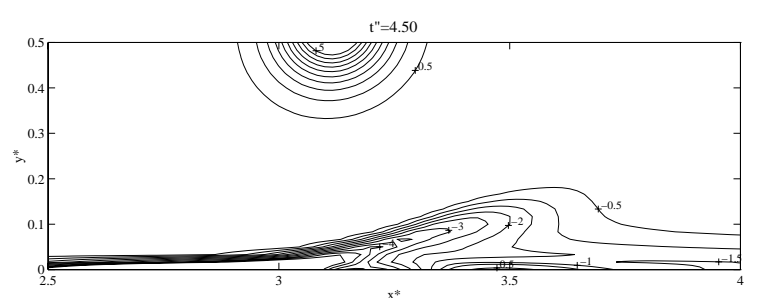

(c) $U_{\max }^{*}=0.10$

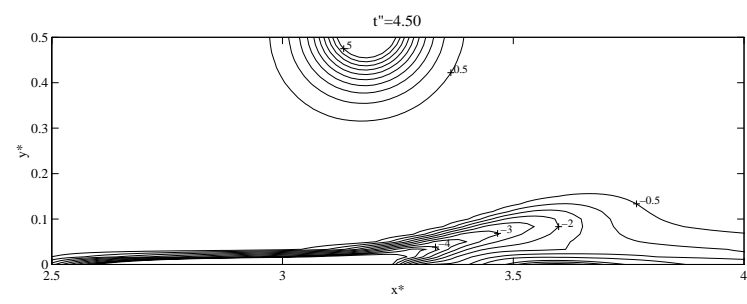

(b) $U_{\max }^{*}=0.05$

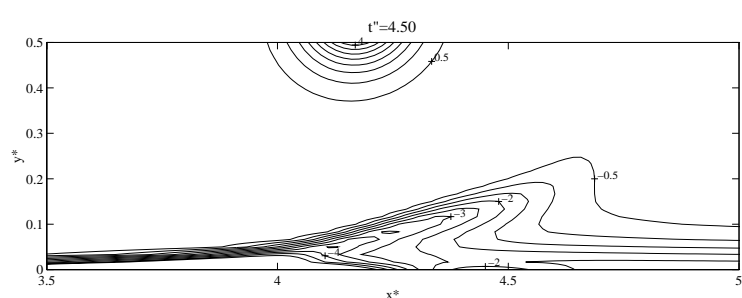

(d) $U_{\max }^{*}=0.20$

Figure 7: Zoom of iso-contours of vorticity for the downwind vortex at $t^{\prime \prime}=4.5$.

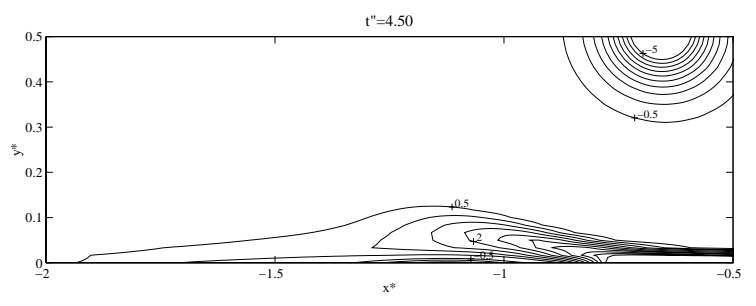

(a) $U_{\max }^{*}=0$

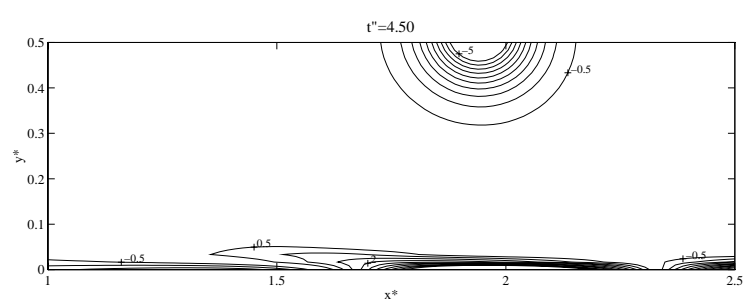

(c) $U_{\max }^{*}=0.10$

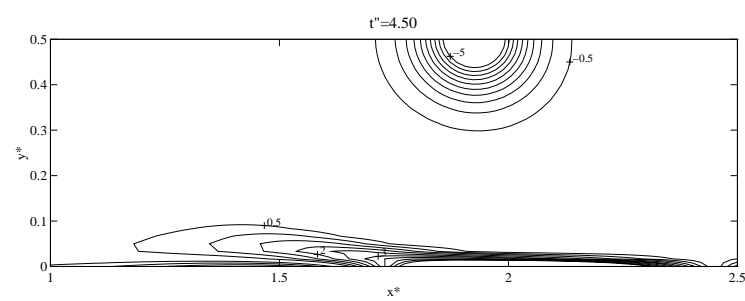

(b) $U_{\max }^{*}=0.05$

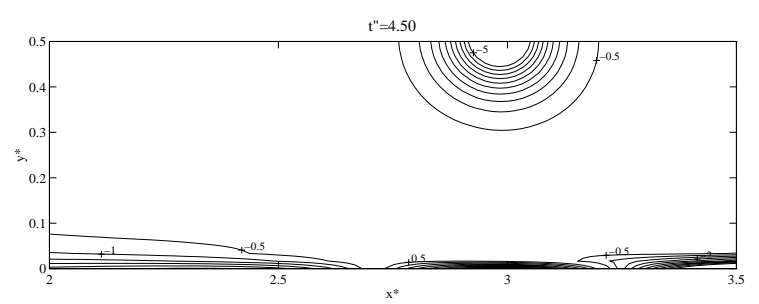

(d) $U_{\max }^{*}=0.20$

Figure 8: Zoom of iso-contours of vorticity for the upwind vortex at $t^{\prime \prime}=4.5$. 


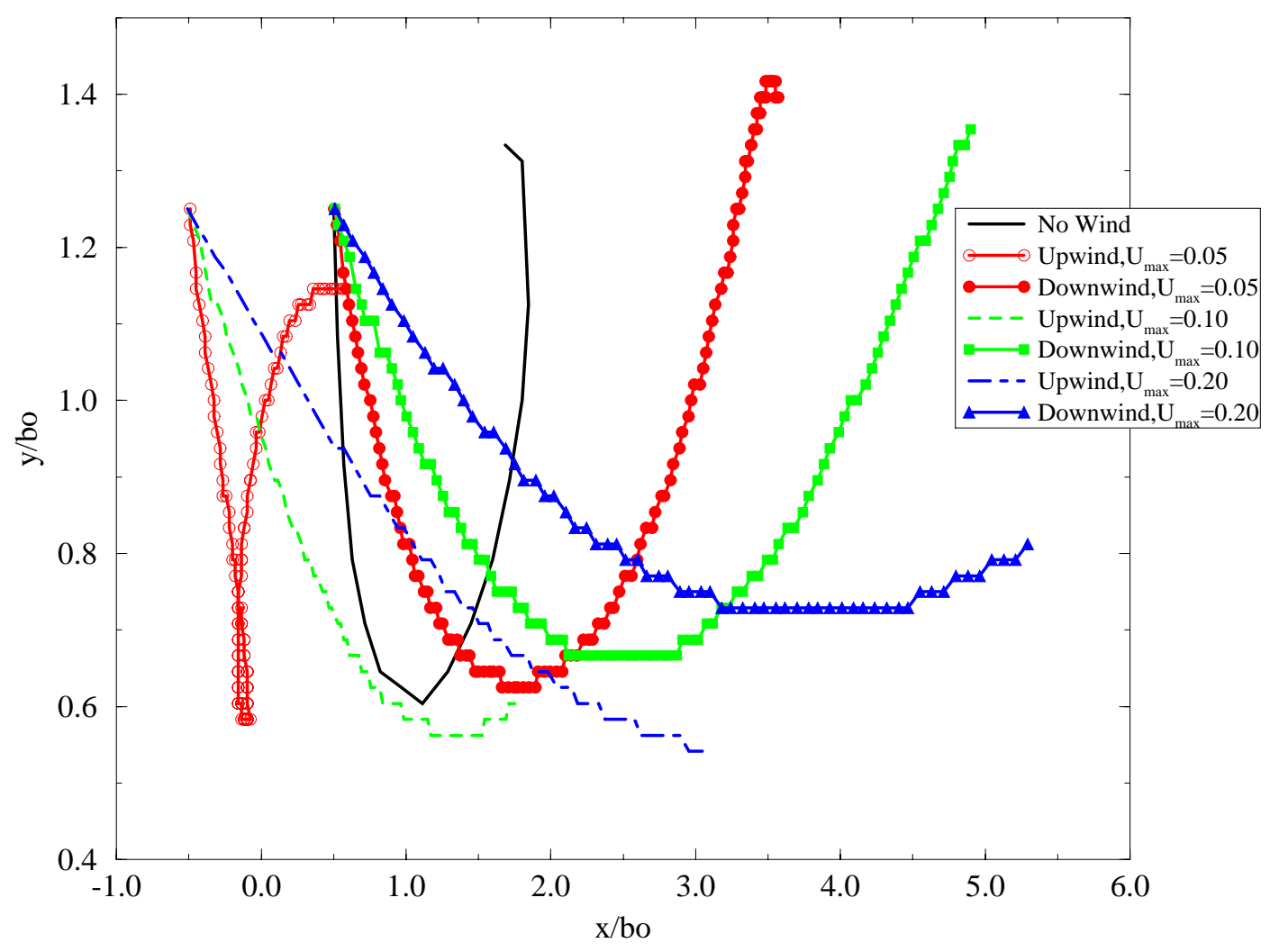

Figure 9: Trajectories. 


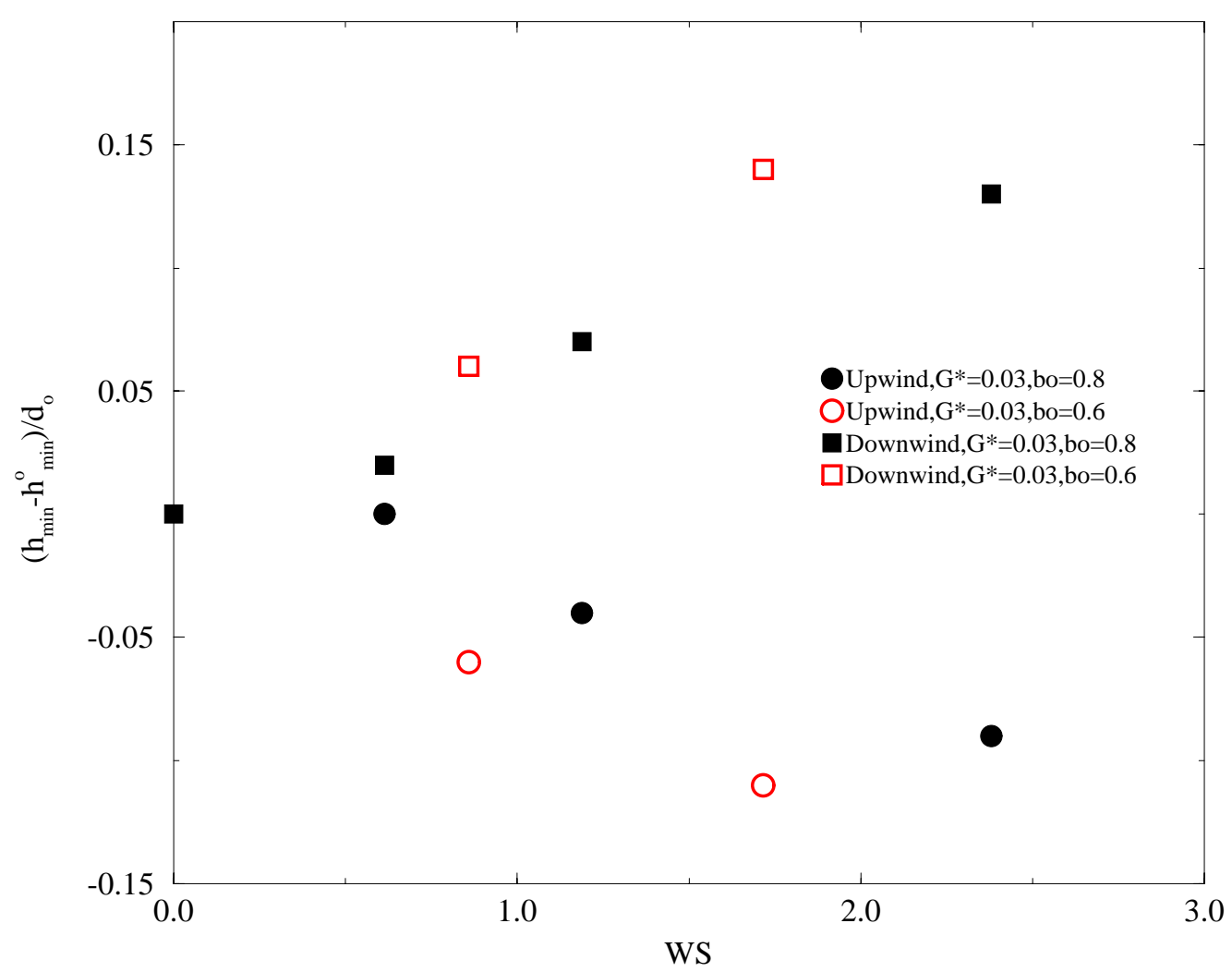

Figure 10: Minimum altitude versus $W_{S}$. 


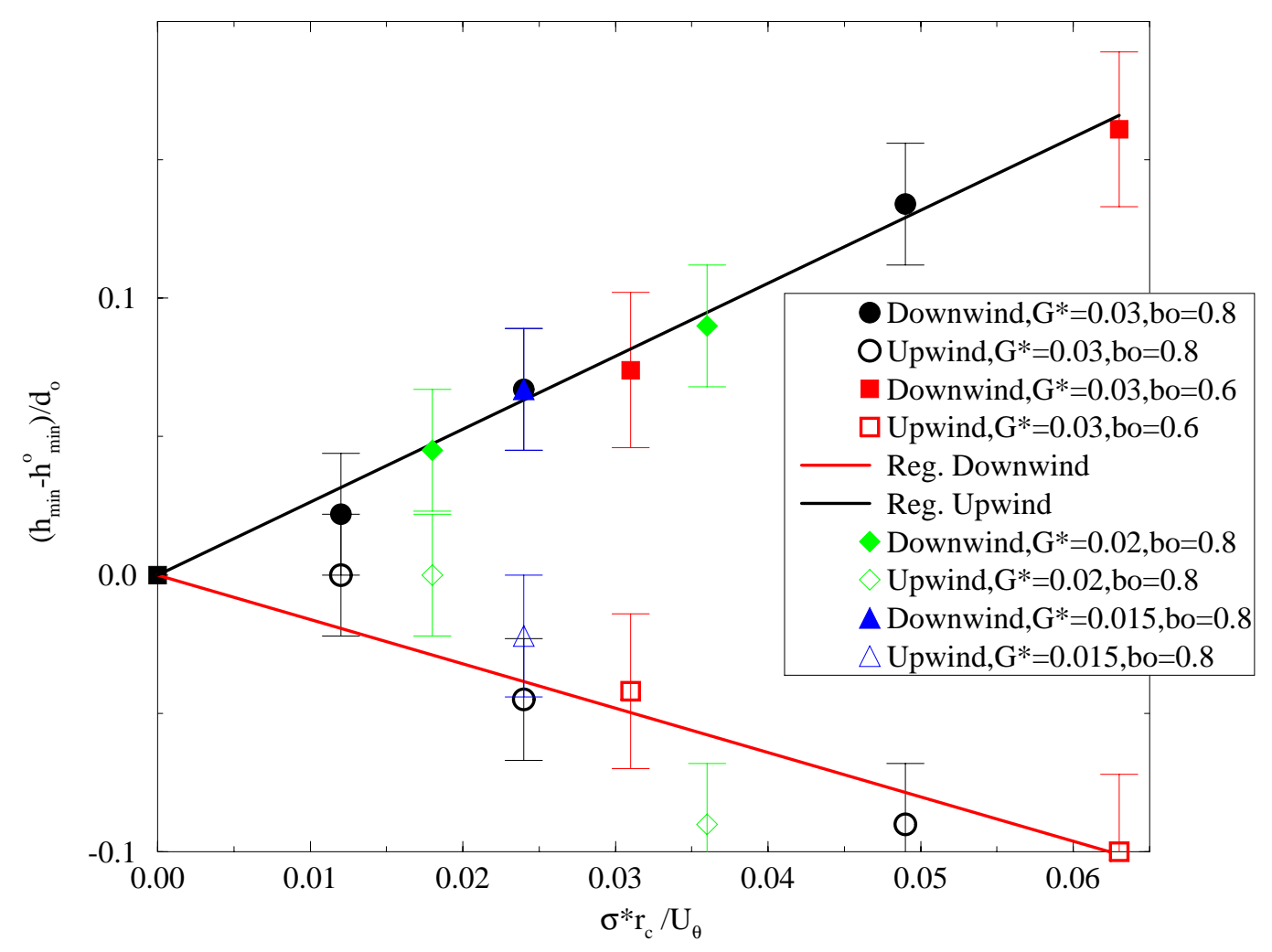

Figure 11: Minimum altitude versus $\sigma / u_{\theta_{\max }} \times r_{c}$.

Table 1: Parameters of the test cases.

\begin{tabular}{lcccccccccccc}
\hline \hline Case & $\frac{b_{0}}{b}$ & $\frac{g}{c b}$ & $\frac{r_{c}}{b}$ & $\frac{U}{c}$ & $\frac{x_{\operatorname{med}}}{b}$ & $\frac{y_{0}}{b}$ & $\frac{d_{0}}{b}$ & $R e_{\text {ref }}$ & $u_{\theta_{\max }}$ & $\frac{u_{\theta_{\max }} r_{c}}{\nu}$ & $\mathrm{Nx}$ & $\mathrm{Ny}$ \\
\hline 1 & 0.8 & 0.03 & 0.1 & 0.00 & 0.0 & 1.0 & 0.74 & 20000 & 0.3 & 600 & 257 & 121 \\
2 & 0.8 & 0.03 & 0.1 & 0.05 & 2.0 & 1.0 & 0.74 & 20000 & 0.3 & 600 & 363 & 121 \\
3 & 0.8 & 0.03 & 0.1 & 0.10 & 1.4 & 1.0 & 0.74 & 20000 & 0.3 & 600 & 363 & 121 \\
4 & 0.8 & 0.03 & 0.1 & 0.20 & 1.4 & 1.0 & 0.74 & 20000 & 0.3 & 600 & 363 & 121 \\
5 & 0.6 & 0.03 & 0.1 & 0.10 & 1.4 & 1.0 & 0.57 & 20000 & 0.3 & 600 & 363 & 121 \\
6 & 0.6 & 0.03 & 0.1 & 0.20 & 1.4 & 1.0 & 0.57 & 20000 & 0.3 & 600 & 363 & 121 \\
7 & 0.8 & 0.02 & 0.1 & 0.05 & 1.4 & 1.0 & 0.74 & 20000 & 0.2 & 400 & 363 & 121 \\
8 & 0.8 & 0.02 & 0.1 & 0.10 & 1.4 & 1.0 & 0.74 & 20000 & 0.2 & 400 & 363 & 121 \\
9 & 0.8 & 0.015 & 0.1 & 0.05 & 1.4 & 1.0 & 0.74 & 20000 & 0.15 & 300 & 363 & 121 \\
\hline \hline
\end{tabular}

